\title{
Electrophysiologic Demonstration of an Atriofascicular Accessory Pathway
}

\author{
WILLIAM H. KOU, FRED MORADY, MICHAEL DE BUITLEIR, \\ and STEVEN D. NELSON \\ From the Divsion of Cardiology, Department of Internal Medicine, University of Michigan \\ Medical Center, Ann Arbor, Michigan
}

\begin{abstract}
KOU, W.H., ET AL.: Electrophysiologic demonstration of an atriofascicular accessory pathway. Evidence of an atriofascicular accessory pathway has not been reported previously. In the patient presented in this case report, an electrophysiology study demonstrated a constant left bundle branch block QRS configuration despite varying degrees of ventricular preexcitation, in association with a constant atrioventricular interval during incremental atrial pacing and programmed atrial stimulation. The presence of an atrioventricular, nodoventricular, nodofascicular, and atrial-His accessory pathway was ruled out. The findings are best explained by the presence of an underlying left bundle branch block and an atriofascicular accessory pathway connecting the atrium and right bundle. The atriofascicular accessory pathway was a passive bystander during an atrial tachycardia and was not directly involved in the generation of the tachycardia. To our knowledge, this is the first report of an atriofascicular accessory pathway. (PACE, Vol. 11, February 1988)
\end{abstract}

atriofascicular accessory pathway, ventricular preexcitation

\section{Introduction}

The existence of atrioventricular and nodoventricular accessory pathways in patients with ventricular preexcitation has been established by electrophysiologic studies. ${ }^{1,2}$ Histologic evidence of atrial-His accessory pathways has been presented $^{3-5}$ and atrial-His accessory pathways have been reported to participate in reentrant tachycardias. ${ }^{6,7}$ However, evidence of an atriofascicular accessory pathway has not been reported previously. We present herein a case in which the electrophysiology study demonstrated the presence of an atriofascicular accessory pathway.

\section{Case Report}

A 62-year-old woman who had an idiopathic dilated cardiomyopathy was referred to the Uni-

Address for reprints: Fred Morady, M.D., Divsion of Cardiology, University Hospital, 1500 East Medical Center Drive, UH B1 F245-0022, Ann Arbor, MI 48109-0022.

Received January 22, 1987; revisions received March 30, 1987 and May 18, 1987; accepted May 19, 1987. versity of Michigan Medical Center for evaluation of paroxysmal palpitations. An electrocardiogram recorded during the palpitations demonstrated a regular, wide QRS tachycardia, which had a left bundle branch block configuration, and a cycle length of $360 \mathrm{~ms}$ (Fig. 1). Treatment with procainamide had been ineffective.

At the time of admission, her blood pressure was $110 / 70$ and her heart rate was $80 /$ minute. Physical examination demonstrated a sustained left ventricular impulse, an $S_{3}$ gallop, and a mitral regurgitation murmur. The chest roentgenogram demonstrated cardiomegaly and mild pulmonary congestion. The electrocardiogram demonstrated a left bundle branch block and a PR interval of 0.20 seconds during sinus rhythm (Fig. 2).

\section{Methods}

After obtaining informed consent, an electrophysiology study was performed in the fasting, unsedated state. Treatment with procainamide was discontinued two days earlier; however, treatment with digoxin, nifedipine, hydralazine, 


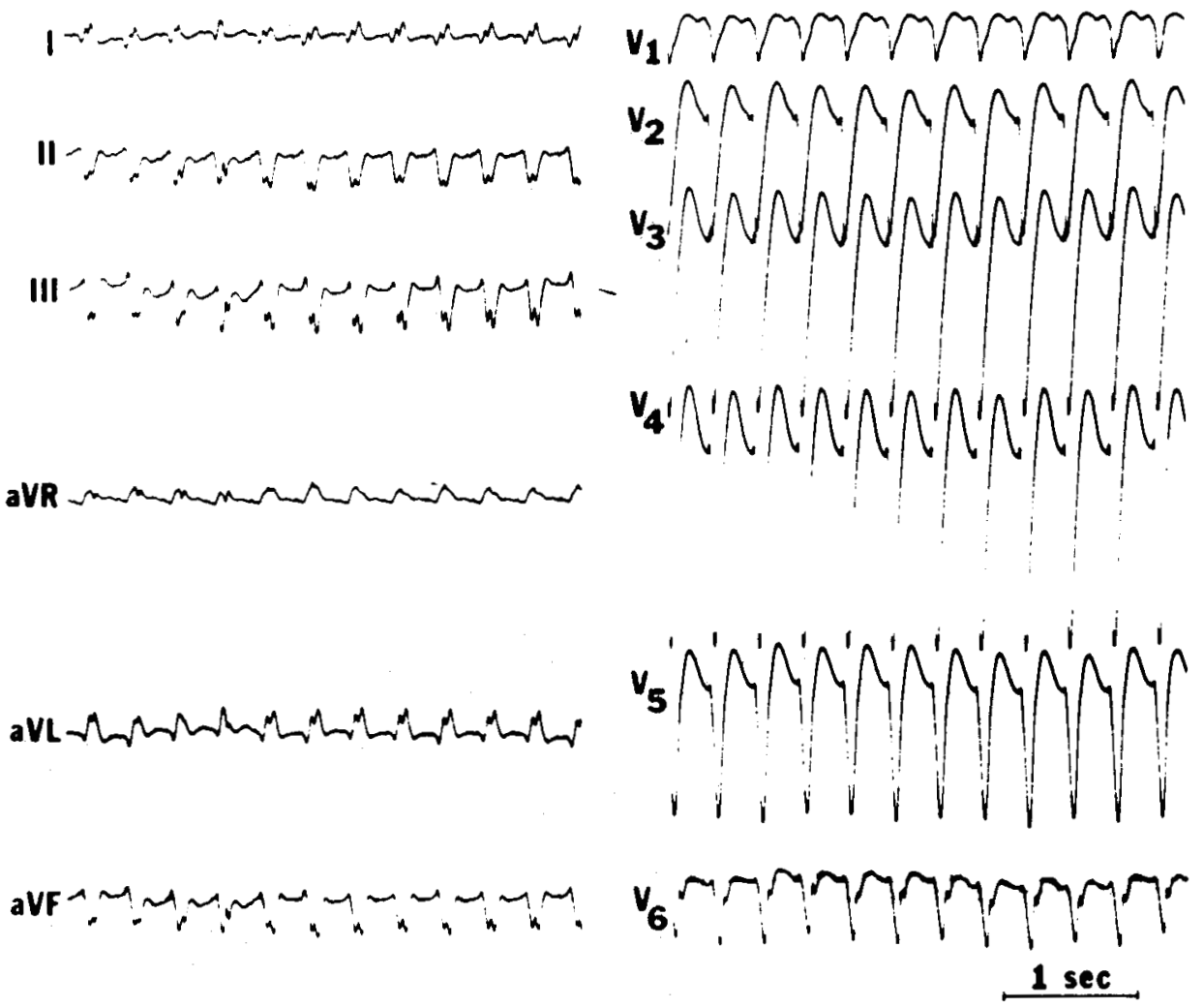

Figure 1. A 12-lead electrocardiographic recording of the patient's tachycardia. The tachycardia has a left bundle branch block configuration and a cycle length of $360 \mathrm{~ms}$.

and bumetanide was continued. Three quadripolar electrode catheters were inserted percutaneously into a femoral vein and positioned against the lateral right atrium, across the tricuspid valve for recording the His bundle electrogram, and against the right ventricular apex. Electrocardiographic leads $V_{1}$, I, and III, the right atrial, His bundle, and right ventricular intracardiac electrograms were displayed on an oscilloscope and recorded at a paper speed of $100 \mathrm{~mm} / \mathrm{s}$ on a Siemens-Elema* Minograf 7 recorder. Programmed stimulation was performed with a programmable stimulator using stimuli $2 \mathrm{~ms}$ in duration and twice the diastolic threshold.

\section{Results}

\section{Baseline Recordings}

Baseline recordings demonstrated a spontaneous sinus cycle length of $760 \mathrm{~ms}$ and a left bun-

* Siemens-Elema, Solna, Sweden. dle branch block. During sinus rhythm, the atrioventricular interval was $125 \mathrm{~ms}$, the atrioventricular nodal conduction time (AH interval) was 80 $\mathrm{ms}$, and the infranodal conduction time (HV interval) was $45 \mathrm{~ms}$ (Fig. 3). An ectopic atrial rhythm was observed frequently, during which the atrioventricular interval was $90 \mathrm{~ms}$, the $\mathrm{AH}$ interval was $80 \mathrm{~ms}$, and the HV interval was $10 \mathrm{~ms}$ (Fig. 3). Of note is that the QRS morphology during the ectopic atrial rhythm was identical to the QRS morphology during sinus rhythm.

\section{Atrial Stimulation}

During incremental right atrial pacing at cycle lengths of 600,500 , and $400 \mathrm{~ms}$, the atrioventricular interval (measured in the His bundle electrogram) remained constant at $130 \mathrm{~ms}$ despite progressive shortening of the HV interval from 45 $\mathrm{ms}$ to $-15 \mathrm{~ms}$ (Fig. 4). At a pacing cycle length of $400 \mathrm{~ms}$, the His depolarization merged with the ventricular electrogram '(Fig. 4). There was no 


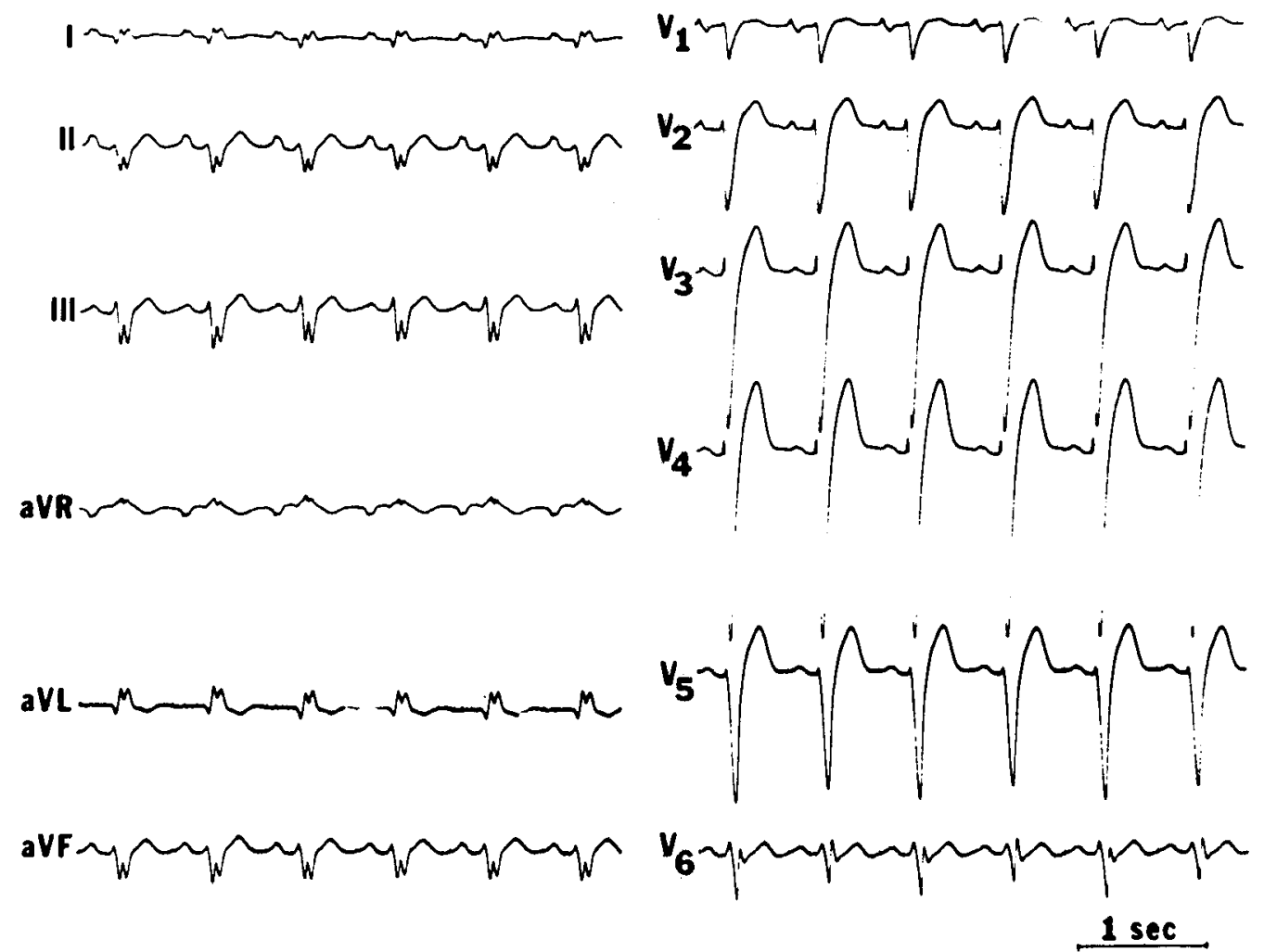

Figure 2. A 12-lead electrocardiographic recording during sinus rhythm. Note that the QRS complexes have the same left bundle branch block configuration present during the tachycardia.

change in the QRS configuration in any of the 12 leads of the electrocardiogram during pacing (Fig. 5). At a pacing cycle length of $350 \mathrm{~ms}$ there was 2:1 atrioventricular block.

At a basic drive cycle length of $670 \mathrm{~ms}$, diastole was scanned with a single atrial extrastimulus. There was gradual lengthening of the AH interval, progressive shortening of the HV interval and no change in the atrioventricular interval or in the QRS configuration. The atrial effective refractory period was $390 \mathrm{~ms}$. The accessory pathway effective refractory period was less than 400 ms. No echo beats were observed.

\section{Ventricular Stimulation}

During incremental right ventricular pacing at cycle lengths of 600 and $500 \mathrm{~ms}$, there was $1: 1$ ventriculoatrial conduction, with a constant ventriculoatrial interval of $240 \mathrm{~ms}$. The low septal right atrial and lateral right atrial electrograms occurred simultaneously. At a ventricular paced cycle length of $450 \mathrm{~ms}$, there was Mobitz II ventriculoatrial block.

Programmed ventricular stimulation at a basic drive cycle length of $600 \mathrm{~ms}$ demonstrated a constant ventriculoatrial interval and a ventricular effective refractory period of $400 \mathrm{~ms}$. The retrograde effective refractory period of the accessory pathway was less than $400 \mathrm{~ms}$.

\section{Tachycardia Characteristics}

Right atrial overdrive pacing at a cycle length of $300 \mathrm{~ms}$ resulted in the induction of a sustained tachycardia which had a cycle length of $360 \mathrm{~ms}$ and the same left bundle branch block configuration which was present during sinus rhythm. The lateral right atrial electrogram preceded the low septal right atrial electrogram during the tachycardia. His bundle depolarizations were not apparent during the tachycardia. Scanning the R-R cycle with a ventricular extrastimulus did not result in premature activation of the atria. 


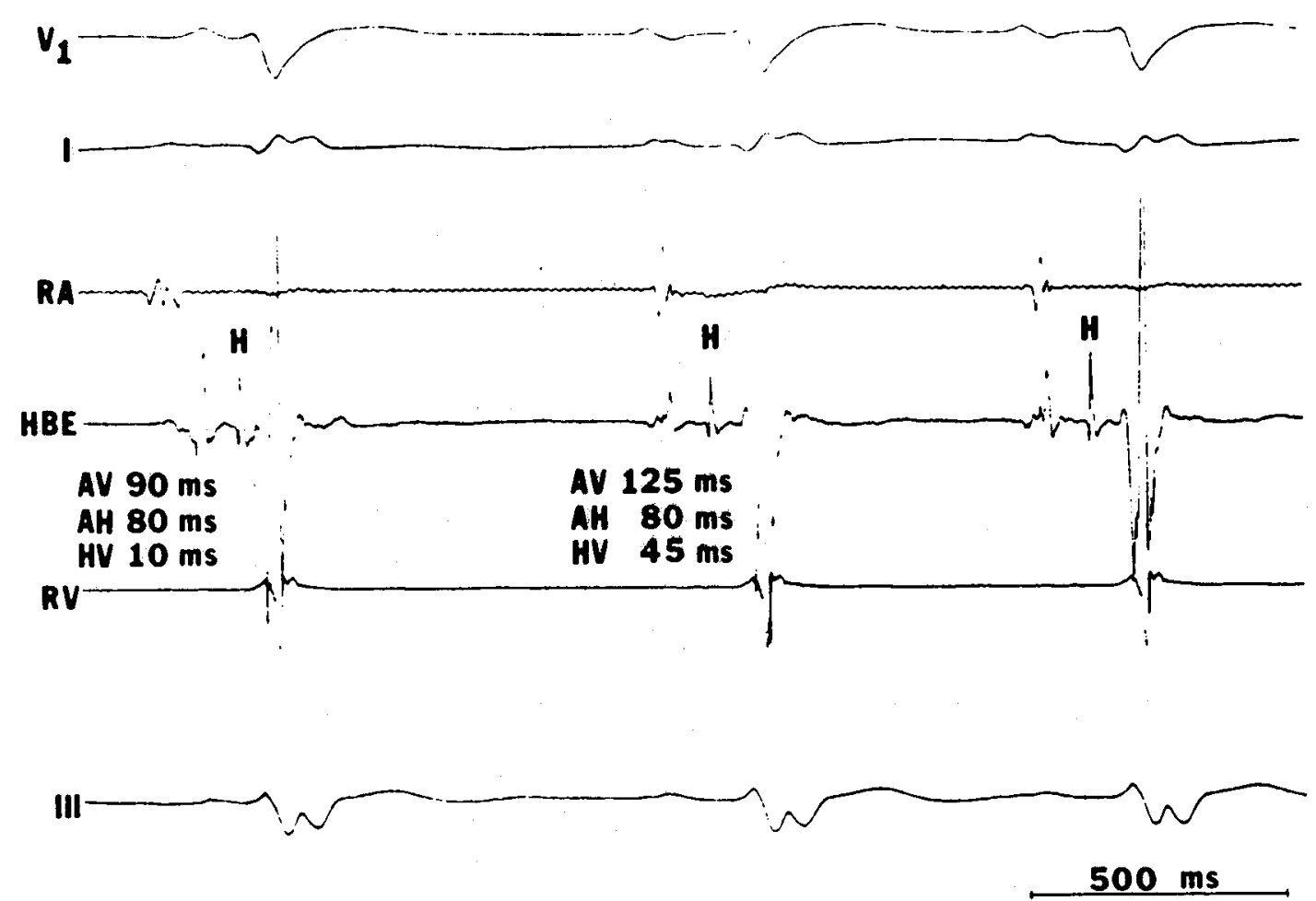

Figure 3. Intracardiac electrograms during sinus rhythm. From top to bottom are leads $V_{1}$ and $I$, an intracardiac recording from the lateral right atrium (RA), the His bundle electrogram (HBE), the right ventricular (RV) electrogram, and lead III. The first $P$ wave arises from an ectopic atrial focus and the next two are sinus beats. Note that the QRS complexes are all identical in configuration, despite differences in the atrioventricular interval (AV) and the infranodal conduction time $(\mathrm{HV}) . \mathrm{AH}=$ atrioventricular nodal conduction time; $\mathrm{H}=$ His bundle depolarization.

The tachycardia could not be terminated by right atrial overdrive pacing. After the intravenous administration of $5 \mathrm{mg}$ of verapamil, the atrial rate did not change, but there was $2: 1$ atrioventricular conduction, with an atrioventricular interval of $80 \mathrm{~ms}$. The His bundle depolarizations were still not apparent.

After $250 \mathrm{mg}$ of intravenous procainamide, the tachycardia was terminated by right atrial pacing.

\section{Characteristics of Junctional Complexes}

Upon termination of the tachycardia, the patient developed a junctional rhythm and atrioventricular dissociation. The cycle length of the ectopic atrial rhythm was $1060 \mathrm{~ms}$ and the cycle length of the junctional rhythm was $620-790 \mathrm{~ms}$. The HV interval of the junctional complexes was $90 \mathrm{~ms}$ and there was no change in the QRS config- uration compared to sinus rhythm (Fig. 6). Upon resumption of conduction during the ectopic atrial rhythm, the HV interval was $15 \mathrm{~ms}$.

\section{Clinical Course}

The patient was treated with flecainide, 150 mg twice daily, after the electrophysiologic study. The paroxysmal tachycardia was responsive to flecainide; however, on the fifth day of therapy, the patient developed acute cardiogenic shock and died. Histologic examination of the cardiac conduction system was not available.

\section{Discussion}

\section{Evidence in Favor of an Atriofascicular Accessory Pathway}

The unique electrophysiologic finding in this case report is that the configuration of the QRS 
KOU, E'T AL.

A $v_{1}$

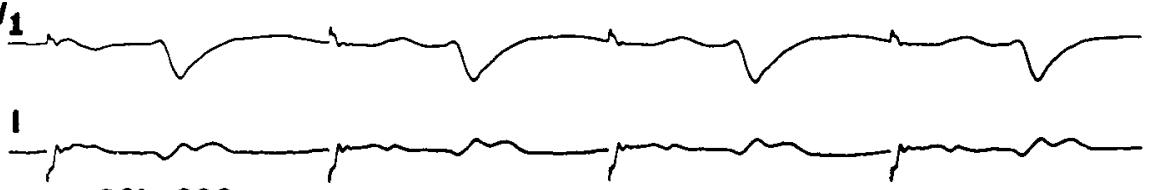

PCL $600 \mathrm{~ms}$

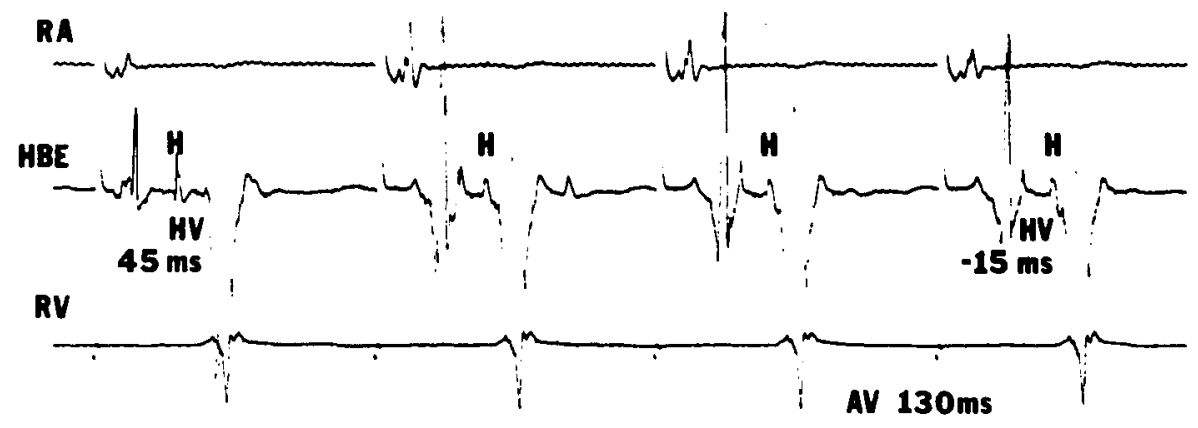

III
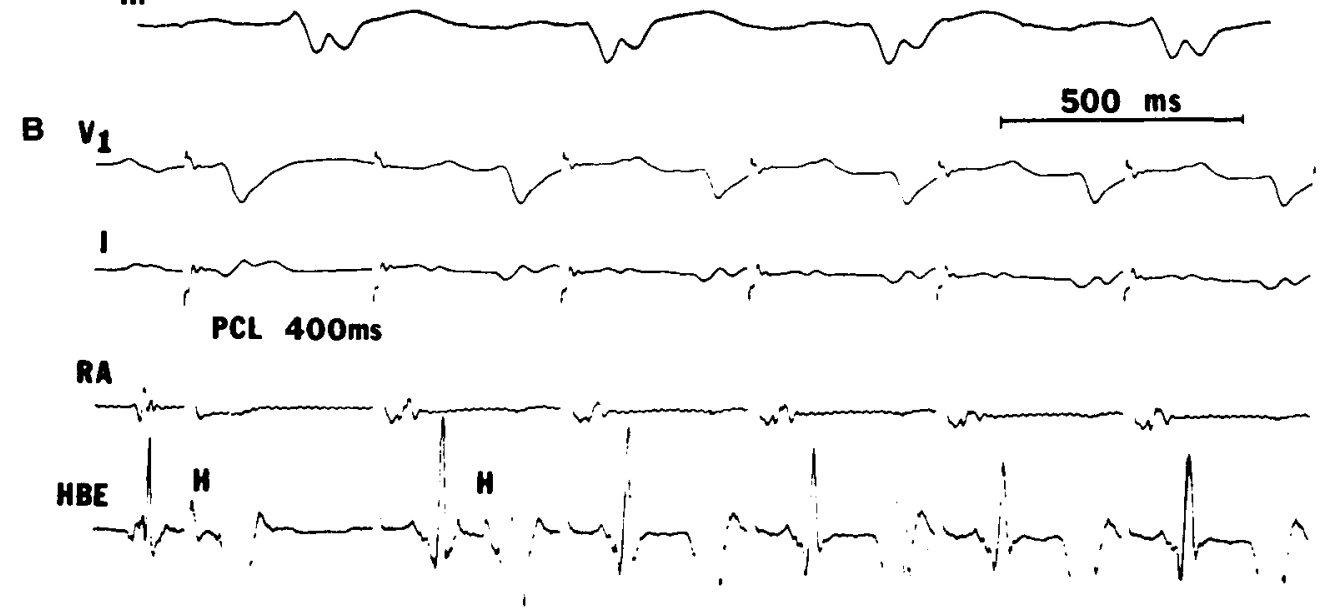

RV
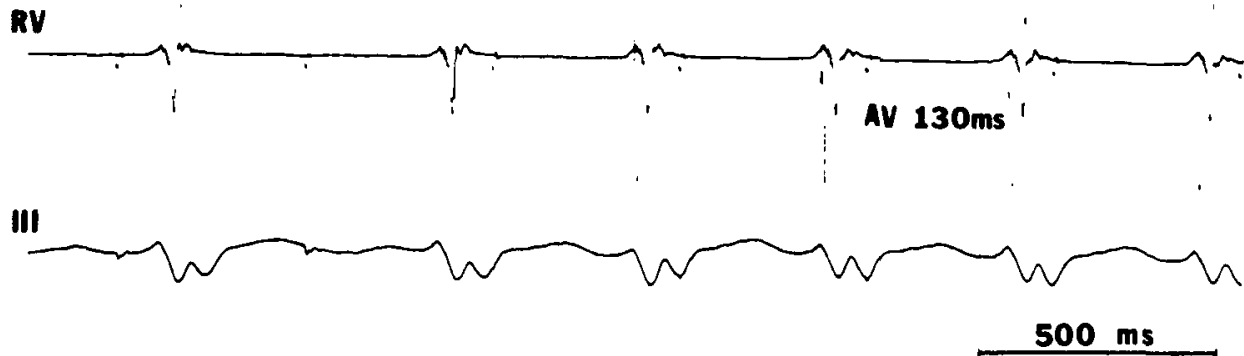

Figure 4. Incremental atrial pacing. A. At a paced cycle length (PCL) of $600 \mathrm{~ms}$, the first stimulus is coincident with a sinus $P$ wave and does not capture the atrium. The next three stimuli capture the right atrium and result in shortening of the $H V$ interval to $-15 \mathrm{~ms}$, with an atrioventricular (AV) interval of $130 \mathrm{~ms}$ (measured in the His bundle electrogram), but no change in the QRS complexes. B. At a PCL of $400 \mathrm{~ms}$, the first stimulus occurs after a sinus $P$ wave and is coincident with the His bundle depolarization. The next five stimuli capture the right atrium. Note that the His bundle depolarization is driven into the ventricular electrogram and is therefore not apparent in the last four complexes. The AV interval is again $130 \mathrm{~ms}$ (measured in the His bundle electrogram) and the QRS complexes are unchanged in configuration. Abbreviations as in Figure 3. 

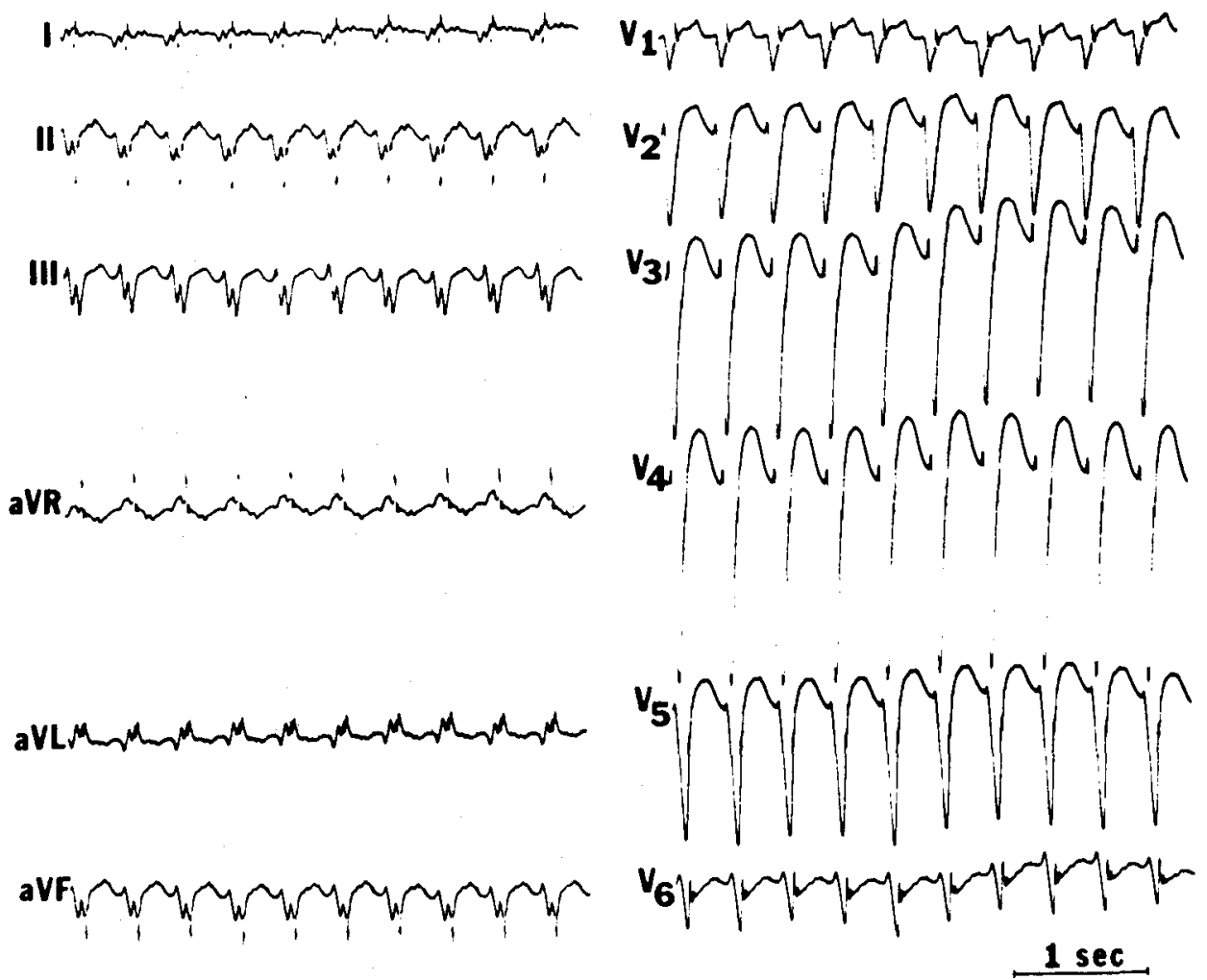

Figure 5. A 12-lead electrocardiographic recording during atrial pacing at a cycle length of 400 ms. The configuration of the QRS complexes is unchanged compared to the QRS configuration during sinus rhythm.

complexes remained unchanged despite varying degrees of ventricular preexcitation. The QRS complexes maintained the same left bundle branch block configuration in association with fluctuation of the HV interval between 45 and $-15 \mathrm{~ms}$, and also when the His bundle depolarization was buried in the ventricular electrogram and not visible. In addition, the atrioventricular interval remained constant during incremental atrial pacing and programmed atrial stimulation.

These observations are best explained by the presence of an underlying left bundle branch block and an atriofascicular accessory connection between the atrium and the right bundle. With an underlying left bundle branch block, it would be expected that the configuration of the QRS complexes would remain unchanged whether ventricular activation occurred predominantly over the atrioventricular node-His-Purkinje axis or the atriofascicular accessory pathway.

\section{Evidence Against Other Types of Accessory Pathways}

An atrioventricular accessory pathway is ruled out by the observation that the configuration of the QRS complexes was identical during sinus rhythm, atrial pacing with varying degrees of ventricular preexcitation, and during junctional rhythm.

Evidence against a nodoventricular or nodofascicular accessory pathway consists of the observation that the atrioventricular interval was constant during incremental atrial pacing and programmed atrial stimulation, and there was a constant ventriculoatrial interval and Mobitz II ventriculoatrial block during incremental ventricular pacing. In addition, a shortening of the AV interval during ectopic atrial rhythm and the loss of ventricular preexcitation during junctional rhythm favor an atrial insertion for the accessory pathway instead of an AV nodal insertion. How- 


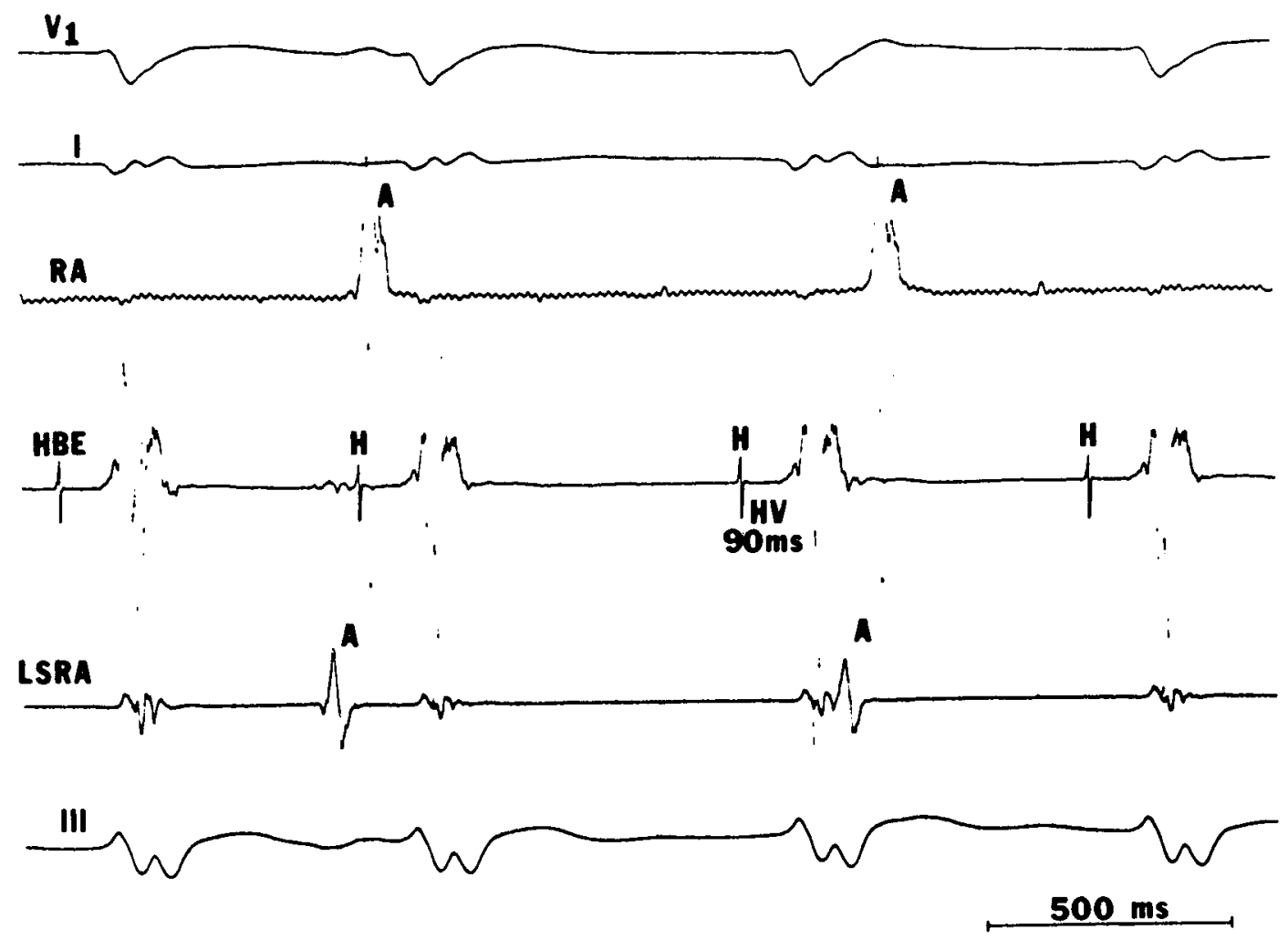

Figure 6. Junctional rhythm and atrioventricular dissociation following the intravenous administration of $5 \mathrm{mg}$ of verapamil and $250 \mathrm{mg}$ of procainamide. The junctional complexes have an $\mathrm{HV}$ interval of $90 \mathrm{~ms}$ and the same left bundle branch block configuration seen during sinus rhythm and atrial pacing. Note that the low septal right atrial electrogram (LSRA) precedes the atrial electrogram recorded at the lateral right atrium (RA). Abbreviations as in Figure 3.

ever, the possibility that the proximal insertion of the accessory pathway was into the upper portion of the AV node cannot be ruled out.

The observation that the HV interval became progressively shorter during incremental atrial pacing and programmed atrial stimulation is not compatible with an atrial-His accessory pathway.

\section{Mechanism of Tachycardia}

Continuation of the tachycardia despite 2:1 atrioventricular block rules out participation of the atriofascicular accessory pathway as a necessary link in a reentrant tachycardia. The observation that the lateral right atrial electrogram preceded the low septal right atrial electrogram during the tachycardia is not compatible with atrioventricular nodal reentrant tachycardia. The characteristics of the tachycardia were compatible with atrial reentry.

\section{Effect of Verapamil}

Two-to-one block in the atriofascicular accessory pathway was induced by verapamil. Although conduction through accessory pathways usually is not affected by calcium channel blocking agents, verapamil has been reported to depress conduction and prolong refractoriness in some atrioventricular accessory pathways. ${ }^{8,9}$ Whether atriofascicular accessory pathways are more often sensitive to calcium channel blocking agents than are other types of accessory pathways is unknown.

\section{Atrial Insertion of the Accessory Pathway}

A limitation of this report is that mapping of the sequence of atrial activation during ventricular pacing was not performed, and therefore the site of atrial insertion of the atriofascicular acces- 


\section{AN ATRIOFASCICULAR ACCESSORY PATHWAY}

sory pathway was not defined. However, based on the shortening in the atrioventricular interval during ectopic atrial rhythm, it is more likely that the atriofascicular accessory pathway inserted into the right atrium than into the left. During the ectopic atrial rhythm, the lateral right atrial electrogram preceded the low septal right atrial electrogram, suggesting that the ectopic atrial rhythm originated in the right atrium. Shortening of the atrioventricular interval during an ectopic right atrial rhythm suggests that the atrial insertion of the atriofascicular accessory pathway was in the right atrium.

\section{References}

1. Gallagher JJ, Pritchett ELC, Sealy WC, et al. The preexcitation syndrome. Prog Cardiovasc Dis 1978; 20:285.

2. Gallagher JJ, Smith WM, Kasell JH, et al. Role of Mahaim fibers in cardiac arrhythmias in man. Circulation 1981; 64:176.

3. Brechenmacher C, Laham J, Iris L, et al. Étude histologique des voies anormales de conduction dans un syndrome de Wolff-Parkinson-White et dans un syndrome de Lown-Ganong-Levine. Arch Mal Coeur 1974; 67:507.

4. Brechenmacher $\mathrm{C}$, Courtadon $\mathrm{M}$, Jourde $\mathrm{M}$, et al. Syndrome de Wolff-Parkinson-White par association de fibres atriohissiennes et de fibres de Mahaim: Confrontation entre l'éléctrophysiologie et l'histologie. Arch Mal Coeur 1976; 69:1275.

5. Brechenmacher $\mathrm{C}$. Atrio-His bundle tracts. $\mathrm{Br}$ Heart J 1975; 37:853-855.

6. Gomes JAC, Dhatt MS, Rubenson DS, et al. Electro-

\section{Conclusion}

In this patient, the accessory pathway was a passive bystander during an atrial tachycardia and was not directly involved in the generation of the tachycardia. Whether atriofascicular accessory pathways are ever directly involved in the generation of reentrant tachycardias remains to be determined.

Acknowledgment: The authors are grateful to Mrs. Lisa Hackbarth for her excellent secretarial assistance.

physiologic evidence for selective retrograde utilization of a specialized conducting system in atrioventricular nodal re-entrant tachycardia. Am J Cardiol 1979; 43:687.

7. Gomes JAC, Dhatt MS, Damato AN, et al. Incidence, determinants and significance of fixed retrograde conduction in the region of the atrioventricular node: Evidence for retrograde atrioventricular nodal bypass tracts. Am J Cardiol 1979; 44:1089.

8. Horio Y, Matsuyama K, Morikami Y, et al. Blocking effect of verapamil on conduction over a catecholamine-sensitive bypass tract in exercise-induced Wolff-Parkinson-White syndrome. J Am Coll Cardiol 1984; 4:186.

9. Tai DY, Chang, MS, Svinarich JT, et al. Mechanisms of verapamil-induced conduction block in anomalous atrioventricular bypass tracts. J Am Coll Cardiol 1985; 5:311. 